\title{
The Fic protein Doc uses an inverted substrate to phosphorylate and inactivate EF-Tu
}

\author{
Daniel Castro-Roa ${ }^{\# 1}$, Abel Garcia-Pino $\# 2,3,{ }^{,}$, Steven De Gieter ${ }^{2,3}$, Nico A.J. van Nuland ${ }^{2,3}$, \\ Remy Loris ${ }^{2,3}$, and Nikolay Zenkin ${ }^{1,{ }^{*}}$ \\ ${ }^{1}$ Centre for Bacterial Cell Biology, Institute for Cell and Molecular Biosciences, Newcastle \\ University, Baddiley-Clark Building, Richardson Road, Newcastle upon Tyne, NE2 4AX, UK \\ ${ }^{2}$ Structural Biology Brussels, Department of Biotechnology (DBIT), Vrije Universiteit Brussel, \\ Pleinlaan 2, B-1050 Brussels, Belgium \\ ${ }^{3}$ Molecular Recognition Unit, Department of Structural Biology, VIB, Pleinlaan 2, B-1050 Brussels, \\ Belgium \\ \# These authors contributed equally to this work.
}

\begin{abstract}
Fic proteins are ubiquitous in all domains of life and play critical roles in multiple cellular processes through AMPylation of (transfer of AMP to) target proteins. Doc from the doc/phd toxin/antitoxin module is a member of the Fic family and inhibits bacterial translation by an unknown mechanism. Here we show that, in contrast to the predicted AMPylating activity, Doc is a new type of kinase that inhibits bacterial translation by phosphorylating the conserved threonine (Thr382) of the translation elongation factor EF-Tu, rendering it unable to bind aminoacylated tRNAs. We provide evidence that EF-Tu phosphorylation diverged from AMPylation by antiparallel binding of the NTP relative to the catalytic residues of the conserved Fic catalytic core of Doc. The results bring insights into the mechanism and role of phosphorylation of EF-Tu in bacterial physiology as well as represent an example of catalytic plasticity of enzymes and a mechanism for the evolution of new enzymatic activities.
\end{abstract}

\section{Introduction}

The Fic (filamentation induced by cyclic AMP) family of enzymes is ubiquitous in all domains of life and is involved in crucial processes, such as bacterial pathogenesis and key signaling events in eukaryotes ${ }^{1-3}$. These proteins are defined by a conserved Fic domain ${ }^{4,5}$ (Figure 1a) that catalyzes AMPylation (also known as adenylylation) of target proteins ${ }^{6}$. It has been suggested that the degeneration of the conserved Fic motif may lead to the gain of a new enzymatic activity or the expected loss of adenylylation activity 1 . Indeed recent reports describing new Fic domains show a departure from the typical purine based NMPylating activity. These domains can catalyze activities as diverse as phosphocholine

\footnotetext{
*Correspondence to: Nikolay Zenkin, PhD Centre for Bacterial Cell Biology Institute for Cell and Molecular Biosciences Newcastle University Baddiley-Clark Building Richardson Road Newcastle upon Tyne NE2 4AX, UK, Phone: +44(0)1912083227, Fax: +44(0)1912083205 n.zenkin@ncl.ac.uk; Abel Garcia-Pino, PhD Structural Biology Brussels Department of Biotechnology Vrije Universiteit Brussel Building E, Pleinlaan 2 Brussels B-1050, Belgium, Phone: +32 (0)2 6291025, Fax: +32 (0)2 6291963 agarciap@vub.ac.be.

Author contributions: D.C-R performed biochemical experiments; S.D.G. prepared Doc and Phd samples; A.G-P prepared Doc and Phd samples, performed NMR and SAXS experiments, analyzed structural data and wrote the paper; N.v.N performed NMR experiments; R.L. analyzed structural data and wrote the paper; N.Z. wrote the paper and supervised the project.

Competing financial interests statement: The authors declare no competing financial interests.
} 
transfer or UMPylation suggesting that catalytic plasticity is a general feature of this family of enzymes ${ }^{7,8}$.

The trademark motif that defines Fic proteins $(\operatorname{HPFx}(\mathrm{D} / \mathrm{E}) \mathrm{GN}(\mathrm{G} / \mathrm{K}) \mathrm{R})$ contains the core residues involved in the catalytic reaction: the transfer of the NMP moiety to the target ${ }^{9}$. Productive binding of the NTP is achieved by correct positioning of the conserved arginine (Arg114 in Figure 1b) of the Fic motif that locks on the ribose ring and the $Y$-phosphate ${ }^{9,10}$. This is accompanied by a direct attack of the hydroxyl group of the side chain of the target protein on the a-phosphate of the nucleotide. The conserved histidine (His107 in Figure 1b) is critical for catalysis as it activates the attacking hydroxyl group via deprotonation ${ }^{9,10}$.

The bacterial toxin Doc is the representative member of a major subfamily of Fic proteins and has a highly conserved Fic catalytic center ${ }^{5,11}$ (Figure 1a, b). Doc belongs to the doc/ phd toxin/antitoxin (TA) module ${ }^{12}$, one of the systems that play a major role in the adaptation of prokaryotes to stress conditions, the persistence phenomenon, programmed cell death and addiction to phages and mobile elements. TA toxins act by inhibiting essential cellular functions to bring the cell to a dormant state, which, for example, becomes temporarily tolerant to antibiotics. The action of TA toxins is blocked in the presence of corresponding antitoxins, the production of which allows the cell to return to normal growth.

Doc has been shown to be a potent inhibitor of bacterial translation ${ }^{5}$. However, its putative adenylylating activity as well as its direct cellular target remains elusive. The current hypothesis proposes that it acts non-catalytically, by sterically obstructing the A-site of ribosomes ${ }^{13}$.

In this paper we show that Doc is a novel type of protein kinase (in contrast with the presumed AMPylating activity or ribosome occlusion ${ }^{11,13}$ ) that inhibits bacterial translation by phosphorylating the conserved threonine $\left(\mathrm{Thr}^{382}\right.$ ) of the translation elongation factor EFTu rendering it unable to bind aminoacylated tRNAs. Our results suggest how this radical switch in catalytic activity is possible despite conservation of the geometry and chemistry of the active center and reveal a remarkable plasticity of the catalytic mechanisms used by the active centers of enzymes.

\section{Results}

\section{Doc inhibits ternary tRNA:EF-Tu:GTP complex formation}

Earlier studies have indicated that Doc inhibits translation in vivo ${ }^{5,13}$. We tested Doc for the ability to inhibit translation in vitro. Doc indeed efficiently inhibited the synthesis of luciferase in a cell-free translation system based on a crude cell extract (Figure 1c). In order to identify the natural target of Doc, we used an in vitro translation system assembled from purified components ${ }^{14}$ (Figure 1d). The mRNA used in this system codes for the initial sequence MFV. Translation is initiated by addition of purified ribosomes, initiation factors IF-1, IF-2, IF-3 and ${ }^{35}$ S]-fMet-tRNA ${ }^{\text {fMet }}$. The ternary complexes (EF-Tu-GTP-PhetRNA $^{\text {Phe }}$ and EF-Tu-GTP-Val-tRNA ${ }^{\mathrm{Val}}$ ) required for translation elongation are prepared separately (Figure 1d). Initiated ribosomes are then allowed to elongate by one (Figure 1e) or two (Figure 1f) codons by addition of preformed ternary complexes and elongation factor EF-G in the presence of GTP. The short peptide products of the reaction are then analyzed by thin layer electrophoresis ${ }^{15}$.

The existing model for the function of Doc suggests that it inhibits translation by blocking the A-site of the ribosome ${ }^{13}$. Unexpectedly, we observed that, when Doc was added to the ribosomes before initiation (Figure 1d), inhibition of peptide synthesis was negligible (Figure 1e). Addition of Doc to the pre-formed ternary complexes (Figure 1d) also had little 
or no effect on translation elongation (Figure 1f). However, addition of Doc before ternary complex formation (the binding event of the aminoacyl-tRNA to the EF-Tu-GTP complex; Figure 1d) resulted in strong inhibition of the synthesis of the dipeptide MF and the tripeptide MFV (Figure 1e, f). These results indicated that Doc does not interfere with tRNA binding to ribosomes, as was suggested earlier ${ }^{13}$ but rather inhibits formation of the ternary tRNA:EF-Tu:GTP complex.

\section{Doc phosphorylates elongation factor EF-Tu}

Given the significant structural similarity of Doc to the Fic domains of AMPylating enzymes $^{5}$ (Figure 1a, b), we hypothesized that Doc might modify one of the components of the ternary complex via a transfer reaction involving GTP present in the reaction mixture for ternary complex formation. Indeed, addition of $\mathrm{Y}\left[{ }^{32} \mathrm{P}\right]-\mathrm{GTP}$ along with Doc to the reaction of ternary complex formation resulted in radiolabelling of EF-Tu (Figure 2a). We also observed EF-Tu radiolabelling by Doc with $y\left[{ }^{32} \mathrm{P}\right]-\mathrm{ATP}$, but not with $\mathrm{a}\left[{ }^{32} \mathrm{P}\right]-\mathrm{ATP}$ or $\mathrm{a}\left[{ }^{32} \mathrm{P}\right]-$ GTP (Figure 2b).

The above results suggested that Doc transferred the $Y$-phosphate (or $\beta / Y$ pyrophosphate) to EF-Tu. This, however, was surprising because most Fic domains (homologs of Doc) catalyze AMPylation of target proteins, i.e. transfer of the AMP- rather than a phosphate moiety. To further investigate which part of the triphosphate moiety of the NTP was transferred to EF-Tu, we analyzed the products of the EF-Tu modification reaction by TLC. Figure 2c shows that incubation of Doc and EF-Tu with $\alpha\left[{ }^{32} \mathrm{P}\right]-\mathrm{ATP}$ resulted in formation of $\mathrm{a}\left[{ }^{32} \mathrm{P}\right]-A D P$. When $y\left[{ }^{32} \mathrm{P}\right]-A T P$ was used in the reaction, we observed accumulation of radiolabeled EF-Tu (which failed to leave the start of the chromatogram; Figure 2c). These results confirmed that Doc phosphorylates EF-Tu by transferring the $\gamma$-phosphate of NTPs. His66 of Doc, an essential residue for AMPylation by Fic proteins, was also critical for Doc function; the H66Y mutant of Doc, while binding EF-Tu with only slightly lower affinity than wild-type Doc (Supplementary Results; Supplementary Table 1), was inactive in phosphorylation (Figure 2d). Consistently, this mutant can be harmlessly expressed in cells without antitoxin ${ }^{16}$. We found that Doc phosphorylated EF-Tu (using either ATP or GTP) at a relatively high rate of $\sim 20 \mathrm{EF}-\mathrm{Tu}$ molecules per second by one molecule of Doc (Supplementary Fig. 2). This is comparable, for example, to the rate of reaction of protein kinase $\mathrm{A}^{17}$. Such a rate of catalysis by Doc, which, in fact, exceeds the rate of amino acid incorporation into the peptide chain ( $\sim 10$ amino acids per second), is consistent with the high toxicity of this protein. Doc phosphorylated EF-Tu efficiently only when added before the aminoacyl-tRNA (Figure 2a), explaining the absence of translation inhibition when Doc was added after ternary complex formation (Figure 1e, f).

LC-MS/MS analysis (after Asp-N and Lys-C digestion of phosphorylated EF-Tu) revealed that Doc phosphorylated EF-Tu at the conserved Thr382 (Supplementary Fig. 4). To confirm these data, we prepared an EF-Tu mutant having Thr328 substituted with Val (EF$\mathrm{Tu}^{\mathrm{T} 382 \mathrm{~V}}$ ). This mutant EF-Tu was active in translation in vitro (Figure 2e), had similar physical characteristics as wild-type EF-Tu (Supplementary Fig. 5a), and had only slightly lower affinity to Doc (Supplementary Table 1). However, Doc did not phosphorylate EF$\mathrm{Tu}^{\mathrm{T} 382 \mathrm{~V}}$ (Figure 2f), supporting our conclusion that Thr382 is the only site on EF-Tu that is phosphorylated by Doc. Furthermore, Doc did not inhibit translation in the presence of the mutant $\mathrm{EF}^{-\mathrm{Tu}^{\mathrm{T}} 382 \mathrm{~V}}$ (Figure 2e), confirming that inactivation of translation by Doc is solely due to the phosphorylation of Thr382 of EF-Tu.

We tested if there were any other targets for phosphorylation by Doc. To do so, we incubated the S30 and S100 fractions of E. coli cell lysate with Doc in the presence of $\mathrm{Y}\left[{ }^{32} \mathrm{P}\right]-\mathrm{ATP}$. The only cell component detectably radiolabeled by Doc was EF-Tu (Figure $2 \mathrm{~g}$ ), suggesting that EF-Tu is likely the sole target for Doc phosphorylation. 


\section{Reversible phosphorylation by Doc}

Most TA toxins catalyze reactions that are irreversible under physiological conditions (e.g. cleavage of mRNA or tRNA ${ }^{18-20}$ ). Their action however, can be counteracted after their inactivation by de novo production of RNA by the transcription machinery. In this context, EF-Tu inactivation by Doc-catalyzed phosphorylation represents an intriguing conundrum. Revival of the cell from the toxin-induced arrested state requires dephosphorylation of EF$\mathrm{Tu}$ in order to rescue the cell from dormancy. However, we did not observe any spontaneous or antitoxin Phd-dependent dephosphorylation of EF-Tu (Figure 3a). In other words, the antitoxin Phd could only block de novo phosphorylation of EF-Tu but could not revert it (Figure 3a, b). Therefore, the revival of the cell likely requires a dedicated phosphatase to dephosphorylate EF-Tu. Interestingly, however, we observed that in the presence of ADP or GDP (the levels of which are likely elevated during dormancy), the phosphorylation of EFTu could be reverted (Figure 3a, c and Supplementary Fig. 6). The dephosphorylation was catalyzed by Doc, as binding of Phd blocked the reaction (Figure 3a). It can be speculated that this Doc-dependent dephosphorylation of EF-Tu may play a physiological role during cell revival from dormancy (see Discussion).

\section{Doc preferentially binds EF-Tu in the GDP-bound state}

The exchange between GTP and GDP bound to EF-Tu acts as an efficient allosteric switch that triggers a dramatic conformational rearrangement in the protein. Binding of GDP exposes a central cavity in the protein and removes the contact between the two individual domains of EF-Tu, leading to the disruption of its tRNA binding site ${ }^{21}$. The GTP-bound state on the other hand is more compact and compatible with tRNA binding ${ }^{22}$. Our isothermal titration calorimetry (ITC) experiments (Supplementary Table 1) showed that Doc preferentially binds to the GDP-bound conformation of EF-Tu. The affinity of Doc for EF-Tu bound to GMPPNP (a non-hydrolysable triphosphate analog of GTP) was about 5fold weaker than the affinity for free EF-Tu and approximately 30 -fold weaker than the affinity for GDP-bound EF-Tu (Supplementary Table 1).

We found that, on its own, Doc binds nucleotides weakly. It had an affinity for AMPPNP in the millimolar range as determined by NMR (Supplementary Table 1). However, when sequestered in the Doc:EF-Tu:GDP complex, the affinity for the AMPPNP increased more than $10^{5}$-fold (Supplementary Table 1). This is a strong indication that several residues from EF-Tu provide extra contacts that complete the nucleotide-binding interface. The Doc:EFTu:GDP complex had a slightly higher affinity for AMPPNP than for GMPPNP (Kd still far below cellular concentrations) but showed no detectable binding to UMPPNP, suggesting that pyrimidine-based nucleotides are poor substrates for the phosphorylation reaction (Supplementary Table 1). As expected, the antitoxin domain of Phd (residues 52-73) completely abrogated binding between Doc and EF-Tu as well as binding of AMPPNP to Doc or to the Doc:EF-Tu:GDP complex (Supplementary Table 1).

\section{Structure of the Doc:EF-Tu:GDP ternary complex}

We used small angle X-ray scattering (SAXS; Figure 4a-e) and NMR chemical shift mapping (Figure 4f-h) to model the structure of the Doc:EF-Tu:GDP complex. The molecular weight of all particles (the Doc:EF-Tu:GDP complex, free EF-Tu and free Doc) determined from the SAXS data agreed well with those predicted from the sequences of both proteins (Supplementary Table 2). Ab initio shape reconstruction of the Doc:EFTu:GDP ternary complex using the program DAMMIF ${ }^{23}$ showed that, compared to the more disk-like shape of EF-Tu in its GDP-bound form ${ }^{21}$, the envelope of the Doc:EF-Tu:GDP complex has a bulky extra density, protruding from the site where Doc is expected to be (Figure 4d, e). 
NMR chemical shift perturbation experiments were used to map the EF-Tu binding site of Doc (Figure 4f-h and Supplementary Table 3). ${ }^{1} \mathrm{H}^{-15} \mathrm{~N}$ HSQC spectrum of ${ }^{13} \mathrm{C}^{15} \mathrm{~N}$-labeled Doc was acquired in the presence and absence of EF-Tu (Figure 4f). The addition of EF-Tu led to small shifts in the resonances of a number of surface residues. The major effect, however, was a reduction of the intensities of Doc cross-peaks (Figure 4g). This effect was likely due to the size of the complex and the exchange between states (free Doc versus the Doc:EF-Tu complex) at rates that cause resonance broadening, as has been described for other interactions of this type ${ }^{24}$. Doc residues exhibiting the largest resonance attenuation by EF-Tu binding clustered together around a shallow groove formed by a-helices $\alpha 2$ and $\alpha 3$ near the N-terminal side of the active site loop (a3-a4) and also near the $\mathrm{N}$-terminal half of a-helix a5 (Figure 4h).

To obtain a pseudo-atomic model of the Doc:EF-Tu:GDP ternary complex starting from their individual atomic coordinates ${ }^{21,25}$ we used the docking protocol implemented in Rosetta ${ }^{26}$ with the chemical shift perturbation data as restraints (see Online Methods, Supplementary Fig. 8 and Supplementary Tables 2 and 3 for further details) and selected the best 100 models (based on the scoring function of the program). We then used CRYSOL ${ }^{27}$ and FoXS ${ }^{28}$ to select from this pool of structures the models with the best agreement with the experimental SAXS scattering curves (Figure 4a-c). Both programs converged in a cluster of structures consistent with the $a b$ initio shapes reconstructed directly from the scattering curve using DAMMIF (Figure 4e; see Online Methods for details). In order to validate our model we mutated Arg64, predicted by SAXS and NMR to be involved in interface contacts, to Gly. ITC confirmed our binding model since the mutation completely disrupted the formation of the Doc:EF-Tu:GDP complex without affecting the overall structure of Doc (Supplementary Fig. 5b, Supplementary Table 1).

In our model of the Doc:EF-Tu:GDP ternary complex Doc occupies the central cavity of EF-Tu facing the loop from the $\beta$-barrel domain III of EF-Tu that contains Thr382 (Figure $4 \mathrm{e}, \mathrm{i})$. Notably, Doc binding site clearly overlaps with the acceptor stem of the tRNA bound to EF-Tu (Figure 4j). The tRNA:EF-Tu:GTP complexes are very stable, and the bound tRNA would occlude the binding site of Doc hindering its binding and precluding EF-Tu phosphorylation. This is fully consistent with our aforementioned observation that Doc is unable to modify EF-Tu once the tRNA:EF-Tu:GTP ternary complex is formed.

\section{Transposed binding of NTP in the active center of Doc}

To further investigate the phenomenon of conversion of AMPylation into phosphorylation activity of the Fic domain of Doc, we performed structural characterization of the Doc-NTP complex. Comparison of the two-dimensional ${ }^{1} \mathrm{H}_{-}{ }^{15} \mathrm{~N}$ HSQC spectra from free and AMPPNP-bound Doc revealed chemical shift perturbations that could be mapped to the surface of the protein (Figure 5a-c). The mapping located the binding epitope for AMPPNP on the deep hydrophobic chasm formed by a-helices $\alpha 1, a 4$ and $a 5$, which extended towards the active site loop (His66 to Arg74) (Figure 5d, Supplementary Fig. 7d and 9). This epitope is distinct from the ATP binding site observed in the crystal structure of Fic from Neisseria meningitidis (NmFic) in complex with AMPPNP ${ }^{1}$ (pdbid entry 3S6A). A docking experiment based on the chemical shift data (see Online Methods and Supplementary Table 3) predicted that the binding of ATP to Doc occurs towards the Cterminal side of the active site loop, whereas, in NmFic, ATP is oriented more towards the $\mathrm{N}$-terminal side of this loop with the nucleoside moiety pointing to the $\mathrm{N}$-terminus of the catalytic His residue (Figure 5d and Supplementary Fig. 9). The latter binding mode would be sterically impossible in Doc because of clashes with a-helix a5 and the presence of the conserved Lys73 in the active site loop of proteins belonging to the Doc family (Supplementary Fig. 9). In typical Fic domains this position is occupied by a glycine, which 
removes the steric hindrance and allows nucleotide binding, and constitutes a major difference in the active site motif between both subfamilies (Supplementary Fig. 9).

In order to validate the orientation of NTP binding by Doc we mutated Asn78 in Doc to Trp. This residue is located on the surface of Doc, in the vicinity of the proposed location of the adenine moiety of AMPPNP but far from the nucleotide-binding site of typical AMPylases. According to CD spectroscopy the mutation did not affect the overall structure of the protein (Supplementary Fig. 5b). ITC showed that the effect of the N78W mutation on the interaction with EF-Tu was negligible (Supplementary Table 1). There was, however, an approximately 100 -fold decrease in the affinity of the Doc ${ }^{\mathrm{N} 78 \mathrm{~W}}$ :EF-Tu:GDP complex for the nucleotide (Supplementary Table 1). This is in agreement with our chemical shift perturbation data and supports the proposed inverted mode of ATP binding.

The antitoxin Phd that inhibits the phosphorylation activity of Doc (Figure 3b) occludes the proposed NTP binding site on Doc ${ }^{5,25}$ (Supplementary Fig. 10). In contrast, if Doc would bind ATP in the classic Fic-like manner, Phd would not obstruct the NTP binding site and its antitoxin function would be severely compromised (Supplementary Fig. 10).

Importantly, in our model, the $\mathrm{Y}$-phosphate of the nucleotide is close to the receiving Thr382 and the catalytic His66 of Doc that activates Thr382 for the nucleophilic attack (see Discussion). Such an inverted orientation of ATP thus allows the transfer of the $Y$-phosphate to EF-Tu instead of the AMP moiety as in the case of classic Fic domains. The inversion of the substrate orientation relative to the catalytic residues with conservation of the geometry of the active site is a highly unusual evolutionary mechanism to generate new enzymatic activities.

\section{Discussion}

Taken together our results revealed that Doc represents a novel class of bacterial protein kinases that modulate bacterial metabolism. Inhibition of translation by Doc is the direct result of phosphorylation of the translation elongation factor EF-Tu without the involvement of the ribosome itself or of any other player of the translation machinery.

Our combined structural and biochemical data allowed us to construct a model for the catalytic activity of Doc that explains the switch from AMPylation to phosphorylation (Figure 6a, b). In the phosphorylation scenario, the active site G/K substitution favors inverted binding of the nucleotide and prevents the NTP binding in the "AMPylation orientation". On the other hand, the chemistry of the active site $(\mathrm{HPFx}(\mathrm{D} / \mathrm{E}) \mathrm{GN}(\mathrm{G} / \mathrm{K}) \mathrm{R})$ remains identical to that of typical Fic-like enzymes. The conserved Arg74 residue locks onto the $\alpha$ - and $\beta$-phosphates of the NTP aided by Lys73, placing the $\gamma$-phosphate in a productive orientation in the proximity of the imidazole group of His66 (Figure 6). NTP hydrolysis then proceeds with the attack of the deprotonated hydroxyl group from EF-TuThr382 which, given the orientation of the NTP in the active site, incorporates the $Y$ phosphate group with the release of the NDP product.

Our data also suggested the mechanism of inhibition of Doc activity by antitoxin Phd. In the complex with Doc, the C-terminal region of antitoxin Phd overlaps with the NTP binding site (Supplementary Fig. 10). Given the high affinity of Phd for $\operatorname{Doc}^{25}$, the antitoxin would easily outcompete the NTP in any scenario with the corresponding inhibitory effect as a result. This observation is also consistent with previous mutagenesis results that show that deletion of the last ten residues of Phd (Phd $\Delta 64-73)$ is sufficient to diminish significantly the antitoxin activity of $\mathrm{Phd}^{29}$. 
Substrate re-orientation in active centers is observed in only some enzyme super-families. In two interrelated families of muconate-lactonizing enzymes, a slight repositioning of the lactone ring with respect to a catalytic Lys defines the stereochemistry of the reaction ${ }^{30}$. In the superfamily of Nudix hydrolases, differences in the motifs surrounding a conserved active center result in a very wide specificity to substrates and hydrolyzed bonds, although there is a considerable variation in the site of attack on the substrate, in the position of the catalytic base and in the number of divalent ions involved ${ }^{31}$. The substrate interconversion in the case of Fic proteins, proposed here, is unusual given that the active center remains conserved, while the reactions involve the transfer of different moieties to protein targets. A similar substrate re-orientation likely takes place in the Fic domain of AnkX, which modulates the activity of Rab GTPases by phosphocholination ${ }^{8}$. Although using CDPcholine as a substrate, it is the phosphocholine moiety that is transferred during the reaction ${ }^{8}$, likely due to antiparallel binding of the substrate. Given the structural similarity between the AnkX domain and Doc, both may have evolved from a common ancestor which had the substrate site transposed compared to common Fic proteins, though convergence of substrate re-orientation is also possible.

A previously proposed model suggests that Doc is a nonspecific inhibitor of translation that interacts with the ribosomal minor subunit and blocks the A-site ${ }^{13}$. This scenario in not consistent with the elevated toxicity and low expression levels of Doc required for inhibition of cell growth and translation under physiological conditions ${ }^{12,25}$. We showed that Doc did not interfere with tRNA binding to the ribosome but directly phosphorylated EF-Tu, resulting in a rapid arrest of translation. Co-purification of Doc with ribosomes as found in an earlier study ${ }^{13}$ is likely caused by association of Doc with ribosomes through EF-Tu. A similar artificial association with ribosomes is observed with toxin VapC, though the function of this toxin is to cleave tRNAs ${ }^{32}$.

We propose that, in the context of TA modules, Doc activity increases the fraction of phosphorylated EF-Tu in the cell ${ }^{33}$ to an extent that is incompatible with efficient translation elongation, thus bringing the cell to a dormant state. It is likely that a specialized phosphatase operates in the cell for EF-Tu dephosphorylation. Our data also suggested that Doc-catalyzed dephosphorylation might play a role in the return from dormancy. Accumulation of NDPs during stasis (in millimolar range as in stationary phase) would result in a baseline level of non-phosphorylated EF-Tu, which may start initial translation upon cell revival. This speculation, however, requires further investigation.

It has been shown in Bacillus subtilis that EF-Tu has a dual function ${ }^{34}$. While only a fraction of EF-Tu is required for maintaining a stable translation rate, a significant portion of the population is involved in the formation of the cytoskeletal structure. The relative amounts of both populations largely depend on the physiological state of the cell with the accumulation of cytoskeletal EF-Tu during stationary phase. The nature of the switch remains an enigma, yet it is tempting to picture a scenario where phosphorylation is the signal to target cytosolic (translation competent) EF-Tu to the more structural function of a cytoskeletal element. In such a scenario the action of Doc would be twofold. On one hand it directly reduces the metabolic pace by inhibition of translation and on the other it would further mimic the stationary phase by accumulating EF-Tu in the cytoskeleton. Indeed it has been observed that upon induction of Doc, cells become appreciably shorter compared to cells growing under normal conditions ${ }^{12}$. At the moment this hypothesis, though fascinating, is in need of thorough validation, and additional experiments are required to further understand the role of phosphorylation in EF-Tu physiology. 


\section{Online Methods}

\section{Protein expression and purification}

Wild type and mutant Doc and Phd have been purified as described ${ }^{25}$. Recombinant wild type and mutant EF-Tu and the remaining components of the pure in vitro translation machinery were produced and purified as described ${ }^{14}$. Commercial cell-free translation system was purchased from Promega.

\section{Inhibition of translation in $E$. coli S30 extract system}

The plasmid plucBEST containing the luciferase gene was used as a reporter in the E. coli S30 extract system (Promega) to test the effect on translation, of $0.1 \mu \mathrm{M}$ Doc. After $30 \mathrm{~min}$ incubation at $37^{\circ} \mathrm{C}$ in the presence of $1 \mu \mathrm{L}$ of $\left[{ }^{35} \mathrm{~S}\right]$-methionine $(10 \mathrm{mCi} / \mathrm{ml}$; Hartmann Analytic) the products were resolved by SDS-PAGE and revealed by phosphorimaging (GE Healthcare). Images of all full gels are shown in Supplementary Fig. 1.

\section{Preparation of ternary complexes}

EF-Tu, aminoacyl-tRNAs and aminoacyl-tRNA synthetases were prepared and purified exactly as described ${ }^{14}$. In order to exchange the GDP bound to purified EF-Tu with GTP, EF-Tu.GDP (400 pmol) was mixed with GTP (600 pmol), phosphoenol pyruvate (800 pmol), and phosphoenol pyruvate kinase $(200 \mu \mathrm{g} / \mathrm{mL})$ with or without Doc (concentration specified in Figures) in $30 \mu \mathrm{L}$ of ternary complex buffer (TcB) $(50 \mathrm{mM}$ Tris- $\mathrm{HCl} \mathrm{pH} \mathrm{7.4,} 40$ $\mathrm{mM} \mathrm{NH} \mathrm{H}_{4} \mathrm{Cl}, 10 \mathrm{mM} \mathrm{MgCl}{ }_{2}$ and $1 \mathrm{mM}$ DTT) at $37^{\circ} \mathrm{C}$ for $10 \mathrm{~min}$. To form the ternary complex EF-Tu-GTP-aminoacyl-tRNA, equimolar amounts of aminoacyl-tRNA (either PhetRNA $^{\text {phe }}$ or Val-tRNA ${ }^{\text {val }}$ were added to the reaction and incubated at $37^{\circ} \mathrm{C}$ for 5 minutes. After that, where stated, Doc was added for $15 \mathrm{~min}$ to the concentrations specified in Figures.

\section{Peptidyl transferase assays}

$70 \mathrm{~S}$ ribosomes, mRNA template, initiation factors, $\left[{ }^{35} \mathrm{~S}\right]-\mathrm{F}-\mathrm{met}_{\mathrm{H}} \mathrm{RNA} \mathrm{fmet}^{\mathrm{fm}}$ and elongation factors were prepared exactly as described ${ }^{14}$. For ribosome binding and translation initiation: $2 \mu \mathrm{M}$ 70S ribosomes were mixed with 2 pmol of mRNA (gene $32-41+81$ relative to the transcriptional start site with the first 3 codons $\mathrm{M}, \mathrm{F}, \mathrm{V}$ ) with or without Doc (concentration specified in the Figures) to a final volume of $49 \mu \mathrm{L}$ of translation buffer (TrLB) $\left(10 \mathrm{mM}\right.$ Tris- $\mathrm{HCl} \mathrm{pH} \mathrm{7,4,60} \mathrm{mM} \mathrm{NH}_{4} \mathrm{Cl}, 10 \mathrm{mM} \mathrm{Mg}(\mathrm{OAc})_{2}$ and $6 \mathrm{mM} \beta-$ mercaptoethanol). The mixture was incubated for 10 minutes at $37^{\circ} \mathrm{C}$ followed by the addition of $5 \mu \mathrm{M}\left[{ }^{35} \mathrm{~S}\right]-F-m e t-t R N A^{\text {fmet }}$, IF-1, IF-2, IF-3 and $200 \mu \mathrm{M}$ GTP to a final volume of $60 \mu \mathrm{L}$. After $10 \mathrm{~min}$ incubation, $10 \mu \mathrm{L}$ aliquots were withdrawn and transferred to tubes containing 1.2X TrLB, $5 \mu \mathrm{M}$ EF-G, $200 \mu \mathrm{M}$ GTP and $6 \mu \mathrm{M}$ ternary complexes for synthesis of MF or MFV. Peptide synthesis was allowed to occur by incubation for 7 minutes at $37^{\circ} \mathrm{C}$. After the elongation reaction, $\mathrm{KOH}$ was added to the final concentration of $100 \mathrm{mM}$ to deacylate the tRNA, and incubated at room temperature for $15 \mathrm{~min}$. The products were resolved by thin layer electrophoresis ${ }^{14}$ and revealed by phosphorimaging (GE Healthcare). Images of full TLEs are shown in Supplementary Fig. 1.

\section{Phosphorylation assays}

EF-Tu phosphorylation was performed in the presence of Doc (concentrations specified in the Figures) and 3 pmol (unless otherwise specified) of $\left[{ }^{32} \mathrm{P}\right]-\mathrm{NTPs}(3000 \mathrm{Ci} / \mathrm{mmol}$; Hartmann Analytic) in TcB. Reactions were incubated for 15 min unless otherwise specified, stopped with Leammli buffer or $200 \mathrm{mM}$ EDTA. Products were resolved by SDSPAGE or TLC using PEI-cellulose developed in $0.5 \mathrm{M} \mathrm{K}_{2} \mathrm{HPO}_{4}$, and revealed by phosphorimaging (GE Healthcare). As mobility standards $1 \mu \mathrm{L}$ of $10 \mathrm{mM}$ NTP or NDP were 
spotted along with the samples, visualized under UV254 light ${ }^{35,36}$, marked with soft pencil and spots of ${ }^{32} \mathrm{P}$ before phosphorimaging. Kinetics data were fitted to a single-exponential equation using SigmaPlot software and normalized to the predicted maximum, which was taken as 100. Images of full TLCs and gels are shown in Supplementary Fig. 1.

\section{Dephosphorylation assays}

The phosphorylation reaction (concentrations specified in Figures) was incubated for $30 \mathrm{~min}$ to ensure transfer of most of $\left[{ }^{32} \mathrm{P}\right]$ onto EF-Tu. After that $1 \mathrm{mM}$ ADP or GDP was added with or without $5 \mu \mathrm{M}$ Phd for 2 hours and the formation of $\mathrm{Y}\left[{ }^{32} \mathrm{P}\right]-\mathrm{ATP}$ or $\mathrm{Y}\left[{ }^{32} \mathrm{P}\right] \mathrm{GTP}$ was monitored by TLC as above. For kinetics analysis, EF-Tu was immobilized in Ni-NTAagarose beads, phosphorylated by Doc with $y\left[{ }^{32} \mathrm{P}\right]$-ATP for $15 \mathrm{~min}$ and then with $1 \mathrm{mM}$ ATP for 15 min to ensure phosphorylation of all EF-Tu. After that beads were washed, and Doc and GDP were added to concentrations and for times as stated in the Figure. The produced $\left.\mathrm{Y}^{[2} \mathrm{P}\right]-\mathrm{GTP}$ was revealed by TLC and phosphorimaging. Kinetics data were fitted to a single-exponential equation using SigmaPlot software and normalized to the predicted maximum, which was taken as 100. Images of full TLCs gels are shown in Supplementary Fig. 1.

\section{Mass Spectrometry}

For the Endo-LysC in-solution digestion $\sim 5$ ug protein sample were dried (in a $0.2 \mathrm{~mL}$ tube), denatured and reduced (by addition of $6 \mathrm{M} \mathrm{GdHCL}$ and $10 \mathrm{mM}$ Tris(2-

carboxyethyl)phosphine respectively) in $0.25 \mathrm{M}$ ammonium bicarbonate $\mathrm{pH} 8$ for $30 \mathrm{~min}$ at $56^{\circ} \mathrm{C}$. The sample was then alkylated with iodoacetamide and reconstituted in $20 \mu \mathrm{l}$ of 25 $\mathrm{mM}$ Tris- $\mathrm{HCl}, \mathrm{pH} 8$ and subsequently digested with $0.05 \mu \mathrm{g}$ sequencing grade endoproteinase LysC (Roche), overnight at $35^{\circ} \mathrm{C}$.

Digested samples were loaded and desalted on a trap column $(0.18 \mathrm{~mm}$ i.d. $\times 5 \mathrm{~mm}$, Waters Symmetry C18) at $15 \mu \mathrm{L} / \mathrm{min}$ with $0.1 \%$ formic acid for $2 \mathrm{~min}$ and then separated on an analytical column $(100 \mu \mathrm{m}$ i.d. $\times 15 \mathrm{~cm}$, Michrom Bioresources Magic C18AQ) over a 30min linear gradient of $5-30 \% \mathrm{CH}_{3} \mathrm{CN}, 0.1 \%$ formic acid. The flow rate through the column was $600 \mathrm{~nL} / \mathrm{min}$. The LTQ FT mass spectrometer was operated in standard data-dependent acquisition mode controlled by the program Xcalibur (Thermo).

LTQ FT MS raw data files were processed to peak lists using the extract_msn.exe tool (Thermo Fisher Scientific). Peak lists were searched using Mascot 2.3 (Matrix Science) using the following parameters: database $=$ NCBInr 20120422 containing the sequence of interest; taxonomy = Escherichia coli; enzyme = semitrypsin; maximum missed cleavages = 2 ; fixed modifications $=$ carbamidomethylation of cysteine; variable modifications $=$ conversion of $\mathrm{N}$-glutamine to pyroglutamate, oxidation of methionine, phosphorylation on $\mathrm{S}, \mathrm{T}$ and $\mathrm{Y}$; parent mass tolerence $=10 \mathrm{ppm}$; fragment mass tolerance $=0.6 \mathrm{Da}$.

The sequence of interest was returned as by far the most significant Mascot match with a protein score of 12085 . The peptides positively identified mapped to $75 \%$ of the sequence coverage and included the site of phosphorylation at Thr-382.

\section{Isothermal titration calorimetry}

All ITC titrations were carried out on an ITC200 apparatus (GE Healthcare). Prior to the measurement, Doc and EF-Tu (and the all their mutants) were dialyzed to $150 \mathrm{mM} \mathrm{NaCl}$, $100 \mathrm{mM}$ sodium phosphate buffer at $\mathrm{pH} 6.5$ or $50 \mathrm{mM}$ Tris- $\mathrm{HCl} \mathrm{pH} \mathrm{7.4,} 40 \mathrm{mM} \mathrm{NH}_{4} \mathrm{Cl}, 10$ $\mathrm{mM} \mathrm{MgCl} 2,1 \mathrm{mM}$ TCEP (and 1mM GDP when appropriate). Nucleotides were directly dissolved into the same buffer and their concentration determined by measurement of their absorption at $259 \mathrm{~nm}$ (AMPPNP), $252 \mathrm{~nm}$ (GMPPNP) and 262nm (UMPPNP). The samples 
were filtered and degassed for $10 \mathrm{~min}$ before being examined in the calorimeter and due to stability reasons the titrations were performed at $5{ }^{\circ} \mathrm{C}$.

We used two different setups for the ITC titrations. In the case of the direct EF-Tu:Doc interaction we titrated EF-Tu into Doc, and, in the cases where excess of nucleotides were used, the same amount of the nucleotide was added to the syringe and the cell of the ITC200 to reduce the contribution of the heat of dilution. For the nucleotide binding, we used a different setup, in this case, with a preformed Doc:EF-Tu:GDP complex in the cell and titrated with the nucleotide (in the syringe of the ITC200). For this type experiments we prepared the complexes starting from free EF-Tu saturated with GDP. The excess GDP was removed by SEC and the EF-Tu:GDP complex was mixed with Doc in equimolar amounts to obtain Doc:EF-Tu:GDP. All the experiments consisted of injection of constant volumes of $2 \mu \mathrm{L}$ of titrant into the cell $(200 \mu \mathrm{L})$ with a stirring rate of $1000 \mathrm{rpm}$. Nominal sample concentrations were between $20 \mu \mathrm{M}$ and $30 \mu \mathrm{M}$ in the cell and $350 \mu \mathrm{M}$ to $500 \mu \mathrm{M}$ in the syringe. Actual sample concentrations were determined after dialysis or buffer exchange by measurement of their absorption at $280 \mathrm{~nm}$.

All data were analyzed using the MicroCal Origin ITC 7.0 software using the 1:1 interaction model. Binding affinities are reported in Supplementary Table 1.

\section{NMR Spectroscopy}

The backbone and side chain assignment of Doc (Supplementary Fig. 7a) was performed as described ${ }^{38}$. A ${ }^{15} \mathrm{~N} /{ }^{13} \mathrm{C}$-labeled sample was prepared for NMR experiments at $500 \mu \mathrm{M}$ in $92 \% \mathrm{H}_{2} \mathrm{O}, 8 \% \mathrm{D}_{2} \mathrm{O}, 150 \mathrm{mM} \mathrm{NaCl}, 100 \mathrm{mM}$ sodium phosphate buffer at $\mathrm{pH} 6.5$ and all the titrations were done in $92 \% \mathrm{H}_{2} \mathrm{O}, 8 \% \mathrm{D}_{2} \mathrm{O}, 150 \mathrm{mM} \mathrm{NaCl}, 100 \mathrm{mM}$ sodium phosphate $\mathrm{pH}$ 6.5 buffer $^{38}$. The backbone and side chain chemical shifts are deposited in the Biological Magnetic Resonance Data Bank (BMRB) under the accession number 1889938. NMR spectra were recorded on Varian NMR Direct-Drive Systems $600 \mathrm{MHz}$ or $800 \mathrm{MHz}$ spectrometers (the latter equipped with a salt-tolerance triple-resonance cold-probe) at $25^{\circ} \mathrm{C}$. All NMR data were processed using NMRPipe ${ }^{39}$ and analyzed with NMRView ${ }^{40}$ and $\mathrm{CCPNMR}^{41}$. For the titration experiments, concentrated ligands (AMPPNP and EF-Tu) were prepared in the same buffer as the protein in the NMR tube. ${ }^{15} \mathrm{~N}^{13} \mathrm{C}_{-}$Doc was used at a concentration of $230 \mu \mathrm{M}$ and the concentrated ligands were added to saturation up to a molar ration of 70 in the case of AMPPNP and 1.5 in case of EF-Tu. AMPPNP was docked onto to Doc (pdbid $3 \mathrm{~K}_{3} 3^{25}$ ) using the protein-ligand protocol implemented in

RosettaLigand ${ }^{42}$ with restraints from the NMR chemical shift mapping. Residues displaying chemical shift differences above $2 \sigma$ (see Supplementary Table 3 and Supplementary Fig. 7d) were used to define the docking interface and searching volume which consisted of a box of dimensions $88 \AA, 86 \AA$ and $66 \AA$. The final model was selected from the 10 best-scoring models as the one satisfying the criteria of having the ligand within $10 \AA$ from each of the residues with chemical shift changes above $2 \sigma$.

\section{Small angle X-ray scattering data collection and modeling}

SAXS data were collected at the PETRA III, P12 beamline (DESY synchrotron, Hamburg), using the standard beamline setup in SEC (size exclusion) mode with the detector (Pilatus $2 \mathrm{M}$ ) at a distance of $3.1 \mathrm{~m}$, and processed with PRIMUS ${ }^{43}$. The samples were prepared in $50 \mathrm{mM}$ Tris- $\mathrm{HCl} \mathrm{pH}$ 7.4, $40 \mathrm{mM} \mathrm{NH}_{4} \mathrm{Cl}, 10 \mathrm{mM} \mathrm{MgCl}_{2}, 1 \mathrm{mM}$ TCEP and $1 \mathrm{mM}$ GDP. The data were analyzed with the ATSAS suite ${ }^{23}$. For the SEC we used a Shodex KW402.5-4F column coupled to an HPLC system, in front of the SAXS data collection capillary, to separate the excess non-complexed material and thus remove this source of background. The sample was passed at a flow rate of $0.2 \mathrm{ml} / \mathrm{min}$ and the data collected at $10^{\circ} \mathrm{C}$. $R g$ values were obtained from the Guinier approximation and the $\mathrm{I}_{\mathrm{o}}$ by extrapolation to $\mathrm{q}=0$, as 
implemented in the ATSAS suite ${ }^{23}$. All the ab initio envelopes were reconstructed from the experimental SAXS data using the program DAMMIF from the ATSAS suite ${ }^{23}$ (see Supplementary Fig. 8 and Supplementary Table 2 for further details on the modeling protocol and validation). In every case the program was run in default mode and the result converted to envelopes using the program Situs ${ }^{44}$.

For modeling the Doc:EF-Tu complex we used a combination of SAXS and docking and the coordinates from Doc (pdbid $3 \mathrm{~K} 33^{25}$ ) and EF-Tu (pdbid $1 \mathrm{EFC}^{21}$ ). The docking experiment was performed using the program Rosetta ${ }^{26}$. In this protocol the program starts 1000 independent simulations from random positions generated from local perturbations of $\sim \pm 3$ $\AA$ in the direction between the two proteins, $\sim 8 \AA$ in the direction of sliding of the proteins relative to each other along their surfaces, $\sim 8^{\circ}$ of tilt of the proteins, and a complete $360^{\circ}$ spin around the axis between the centers of the two proteins. The initial search region was defined using the chemical shift perturbation information ${ }^{26}$. To guide the docking we restrained to a maximum of $10 \AA$ the distance between T382 of EF-Tu and residues S27, R31 and R64 of Doc (all displaying chemical shift changes above $2 \sigma$ ) and to a maximum of $5 \AA$ the catalytic H66 that de-protonates T382. These constraints were implemented in Rosetta using the BOUNDED scoring function. The best 100 models based on the scoring function of the program were then used as input for $\mathrm{CRYSOL}^{27}$ and $\mathrm{FoXS}^{28}$ to select from this pool of structures the models with the best agreement with the experimental SAXS scattering curves. Solutions with $x^{2}$ below 1.1 (15 models) were clustered in three groups with a core r.m.s.d below $1.5 \AA$ over $510 \mathrm{Ca}$ atoms. These models could be fitted to the experimental data with $\mathrm{X}^{2}$ between 0.9 and 1.1 (Supplementary Fig. 8 e-g). They are also in agreement with the other residues in Doc with chemical shift changes above $2 \sigma$, which were not used as restraints. The quality of the models was assessed based on the comparison with the experimental data through the $x^{2}$ and $\mathrm{R}_{\mathrm{SAS}}{ }^{45}$. In every case there is an excellent agreement to the experimental data and the fitting parameters of the models to the data are within acceptable values ${ }^{45}$.

\section{Small angle X-ray scattering data validation}

In order to validate the quality of the SAXS-based model we used the metrics proposed recently ${ }^{45}$ which make use of the $\mathrm{q}(\mathrm{I}(\mathrm{q}))$ v.s. q invariant discovered by the authors. In their work they suggest that SAXS based models that fit the criteria of $x^{2}<1.5$ and $\mathrm{R}_{\mathrm{SAS}}<0.005$ (equation 2) possess acceptable quality for the selected resolution cutoff.

In every case the quality of our models is in excellent agreement with the experimental data and the fitting parameters of the models to the data are within acceptable values ${ }^{45}$ (see Supplementary Table 2).

$$
\mathrm{Vc}=\frac{\mathrm{v}_{\mathrm{p}}}{2 \pi \mathrm{l}_{\mathrm{c}}} \quad \text { equation } 1
$$

with $V p$ as the Porod volume

$$
R_{S A S}=\frac{\left(R_{g}^{\text {exp }}-R_{g}^{\text {model }}\right)^{2}}{\left(R_{g}^{\text {exp }}\right)^{2}}+\frac{\left(V_{c}^{\text {exp }}-V_{c}^{\text {model }}\right)^{2}}{\left(V_{c}^{\text {exp }}\right)^{2}} \text { equation 2 }
$$

\section{Supplementary Material}

Refer to Web version on PubMed Central for supplementary material. 


\section{Acknowledgments}

We thank Robert van Nues and David Forrest for critical reading of the manuscript, Ariel Talavera for assistance with the docking experiments and Joe Gray for support with mass spectrometry. This work was supported by UK Biotechnology and Biological Sciences Research Council and the European Research Council [ERC-2007-StG 202994-MTP] to NZ, the Onderzoeksraad of the VUB, FWO-Vlaanderen, VIB and the Hercules Foundation. The authors acknowledge the use of the EMBL beamline P12 at the DESY synchrotron (Hamburg, Germany).

\section{References}

1. Engel P, et al. Adenylylation control by intra- or intermolecular active-site obstruction in Fic proteins. Nature. 2012; 482:107-10. [PubMed: 22266942]

2. Woolery AR, Luong P, Broberg CA, Orth K. AMPylation: Something Old is New Again. Front Microbiol. 2010; 1:113. [PubMed: 21607083]

3. Yarbrough ML, Orth K. AMPylation is a new post-translational modiFICation. Nat Chem Biol. 2009; 5:378-9. [PubMed: 19448605]

4. Anantharaman V, Aravind L. New connections in the prokaryotic toxin-antitoxin network: relationship with the eukaryotic nonsense-mediated RNA decay system. Genome Biol. 2003; 4:R81. [PubMed: 14659018]

5. Garcia-Pino A, et al. Doc of prophage P1 is inhibited by its antitoxin partner Phd through fold complementation. J Biol Chem. 2008; 283:30821-7. [PubMed: 18757857]

6. Yarbrough ML, et al. AMPylation of Rho GTPases by Vibrio VopS disrupts effector binding and downstream signaling. Science. 2009; 323:269-72. [PubMed: 19039103]

7. Feng F, et al. A Xanthomonas uridine $5^{\prime}$-monophosphate transferase inhibits plant immune kinases. Nature. 2012; 485:114-8. [PubMed: 22504181]

8. Mukherjee S, et al. Modulation of Rab GTPase function by a protein phosphocholine transferase. Nature. 2011; 477:103-6. [PubMed: 21822290]

9. Xiao J, Worby CA, Mattoo S, Sankaran B, Dixon JE. Structural basis of Fic-mediated adenylylation. Nat Struct Mol Biol. 2010; 17:1004-10. [PubMed: 20622875]

10. Luong P, et al. Kinetic and structural insights into the mechanism of AMPylation by VopS Fic domain. J Biol Chem. 2010; 285:20155-63. [PubMed: 20410310]

11. Kinch LN, Yarbrough ML, Orth K, Grishin NV. Fido, a novel AMPylation domain common to fic, doc, and AvrB. PLoS One. 2009; 4:e5818. [PubMed: 19503829]

12. Lehnherr H, Maguin E, Jafri S, Yarmolinsky MB. Plasmid addiction genes of bacteriophage P1: doc, which causes cell death on curing of prophage, and phd, which prevents host death when prophage is retained. J Mol Biol. 1993; 233:414-28. [PubMed: 8411153]

13. Liu M, Zhang Y, Inouye M, Woychik NA. Bacterial addiction module toxin Doc inhibits translation elongation through its association with the $30 \mathrm{~S}$ ribosomal subunit. Proc Natl Acad Sci U S A. 2008; 105:5885-90. [PubMed: 18398006]

14. Castro-Roa D, Zenkin N. In vitro experimental system for analysis of transcription-translation coupling. Nucleic Acids Res. 2012; 40:e45. [PubMed: 22210860]

15. Brunelle JL, Youngman EM, Sharma D, Green R. The interaction between C75 of tRNA and the A loop of the ribosome stimulates peptidyl transferase activity. RNA. 2006; 12:33-9. [PubMed: 16373492]

16. Garcia-Pino A, et al. Crystallization of Doc and the Phd-Doc toxin-antitoxin complex. Acta Crystallogr Sect F Struct Biol Cryst Commun. 2008; 64:1034-8.

17. Shaffer J, Adams JA. Detection of conformational changes along the kinetic pathway of protein kinase A using a catalytic trapping technique. Biochemistry. 1999; 38:12072-9. [PubMed: 10508411]

18. Vesper O, et al. Selective translation of leaderless mRNAs by specialized ribosomes generated by MazF in Escherichia coli. Cell. 2011; 147:147-57. [PubMed: 21944167]

19. Winther KS, Gerdes K. Enteric virulence associated protein VapC inhibits translation by cleavage of initiator tRNA. Proc Natl Acad Sci U S A. 2011; 108:7403-7. [PubMed: 21502523] 
20. Yamaguchi Y, Inouye M. mRNA interferases, sequence-specific endoribonucleases from the toxinantitoxin systems. Prog Mol Biol Transl Sci. 2009; 85:467-500. [PubMed: 19215780]

21. Song H, Parsons MR, Rowsell S, Leonard G, Phillips SE. Crystal structure of intact elongation factor EF-Tu from Escherichia coli in GDP conformation at 2.05 A resolution. J Mol Biol. 1999; 285:1245-56. [PubMed: 9918724]

22. Nissen P, et al. Crystal structure of the ternary complex of Phe-tRNAPhe, EF-Tu, and a GTP analog. Science. 1995; 270:1464-72. [PubMed: 7491491]

23. Konarev PV, Petoukhov MV, Volkov VV, Svergun DI. ATSAS 2.1, a program package for smallangle scattering data analysis. J. Appl. Cryst. 2006; 39:277-286.

24. Park SJ, Borin BN, Martinez-Yamout MA, Dyson HJ. The client protein p53 adopts a molten globule-like state in the presence of Hsp90. Nat Struct Mol Biol. 2011; 18:537-41. [PubMed: 21460846]

25. Garcia-Pino A, et al. Allostery and intrinsic disorder mediate transcription regulation by conditional cooperativity. Cell. 2010; 142:101-11. [PubMed: 20603017]

26. Gray JJ, et al. Protein-protein docking with simultaneous optimization of rigid-body displacement and side-chain conformations. J Mol Biol. 2003; 331:281-99. [PubMed: 12875852]

27. Lehnherr H, Yarmolinsky MB. Addiction protein Phd of plasmid prophage P1 is a substrate of the ClpXP serine protease of Escherichia coli. Proc Natl Acad Sci U S A. 1995; 92:3274-7. [PubMed: 7724551]

28. Schneidman-Duhovny D, Hammel M, Sali A. FoXS: a web server for rapid computation and fitting of SAXS profiles. Nucleic Acids Res. 2010; 38:W540-4. [PubMed: 20507903]

29. McKinley JE, Magnuson RD. Characterization of the Phd repressor-antitoxin boundary. J Bacteriol. 2005; 187:765-70. [PubMed: 15629948]

30. Sakai A, et al. Evolution of enzymatic activities in the enolase superfamily: stereochemically distinct mechanisms in two families of cis,cis-muconate lactonizing enzymes. Biochemistry. 2009; 48:1445-53. [PubMed: 19220063]

31. Mildvan AS, et al. Structures and mechanisms of Nudix hydrolases. Arch Biochem Biophys. 2005; 433:129-43. [PubMed: 15581572]

32. Winther KS, Gerdes K. Regulation of enteric vapBC transcription: induction by VapC toxin dimerbreaking. Nucleic Acids Res. 2012; 40:4347-57. [PubMed: 22287572]

33. Lippmann C, et al. Prokaryotic elongation factor Tu is phosphorylated in vivo. J Biol Chem. 1993; 268:601-7. [PubMed: 8416965]

34. Defeu Soufo HJ, et al. Bacterial translation elongation factor EF-Tu interacts and colocalizes with actin-like MreB protein. Proc Natl Acad Sci U S A. 2010; 107:3163-8. [PubMed: 20133608]

35. Cusack NJ, Pearson JD, Gordon JL. Stereoselectivity of ectonucleotidases on vascular endothelial cells. Biochem J. 1983; 214:975-81. [PubMed: 6312968]

36. Lycan DE, Danna KJ. Characterization of the 5 ' termini of purified nascent simian virus 40 late transcripts. J Virol. 1983; 45:264-74. [PubMed: 6296435]

37. Boon $\mathrm{K}$, et al. Isolation and functional analysis of histidine-tagged elongation factor Tu. Eur J Biochem. 1992; 210:177-83. [PubMed: 1446670]

38. De Gieter S, Loris R, van Nuland NA, Garcia-Pino A. (1)H, (13)C, and (15)N backbone and sidechain chemical shift assignment of the toxin Doc in the unbound state. Biomol NMR Assign. 2013

39. Delaglio F, et al. NMRPipe: a multidimensional spectral processing system based on UNIX pipes. J Biomol NMR. 1995; 6:277-93. [PubMed: 8520220]

40. Johnson BA. Using NMRView to visualize and analyze the NMR spectra of macromolecules. Methods Mol Biol. 2004; 278:313-52. [PubMed: 15318002]

41. Vranken WF, et al. The CCPN data model for NMR spectroscopy: development of a software pipeline. Proteins. 2005; 59:687-96. [PubMed: 15815974]

42. Meiler J, Baker D. ROSETTALIGAND: protein-small molecule docking with full side-chain flexibility. Proteins. 2006; 65:538-48. [PubMed: 16972285]

43. Konarev PV, Volkov VV, Sokolova AV, Koch MHJ, Svergun DI. PRIMUS: a Windows PC-based system for small-angle scattering data analysis. J. Appl. Cryst. 2003; 36:1277-1282. 
44. Wriggers W. Using Situs for the integration of multi-resolution structures. Biophys Rev. 2010; 2:21-27. [PubMed: 20174447]

45. Rambo RP, Tainer JA. Accurate assessment of mass, models and resolution by small-angle scattering. Nature. 2013; 496:477-81. [PubMed: 23619693] 



Figure 1. Doc inhibits translation by inactivation of ternary complex formation (a). Ribbon representation of the structural superposition of Doc (pdbid $3 \mathrm{~K} 33^{25}$ ) onto Fic adenylylase from $N$. meningitides (NmFic; pdbid $3 \mathrm{~S}^{2} \mathrm{~A}^{1}$ ). Doc is colored in blue and NmFic in pink, with respective catalytic loops in light and dark green. (b). Superposition of the catalytic loops of Doc (residues H66-R74) and NmFic (in the nucleotide bound conformation; residues H107-R114). The AMPPNP molecule bound to NmFic is shown in black (c). Luciferase synthesis in a commercially available cell-free translation system was performed in the presence of $\left[{ }^{35} \mathrm{~S}\right]$-methionine in the absence or presence of Doc and revealed by phosphorimaging. Here and after, full images of gels, TLCs and TLEs are presented in Supplementary Fig. 1. (d). Scheme of the assembly of an in vitro translation system using purified components (PK-pyruvate kinase; PEP-phosphoenol pyruvate). Steps at which Doc was added to the reactions in panels (e) and (f) and in Figure 2a, e are depicted in colors. (e) Synthesis of dipeptide MF in the absence or presence of Doc added after (red) or during (blue) ternary complex (TC) formation. (f). Synthesis of tripeptide MFV in the presence or absence of Doc added during initiation (cyan) or ternary (blue) complex formation. Peptides were analyzed by thin layer electrophoresis and autoradiography ${ }^{14}$. 

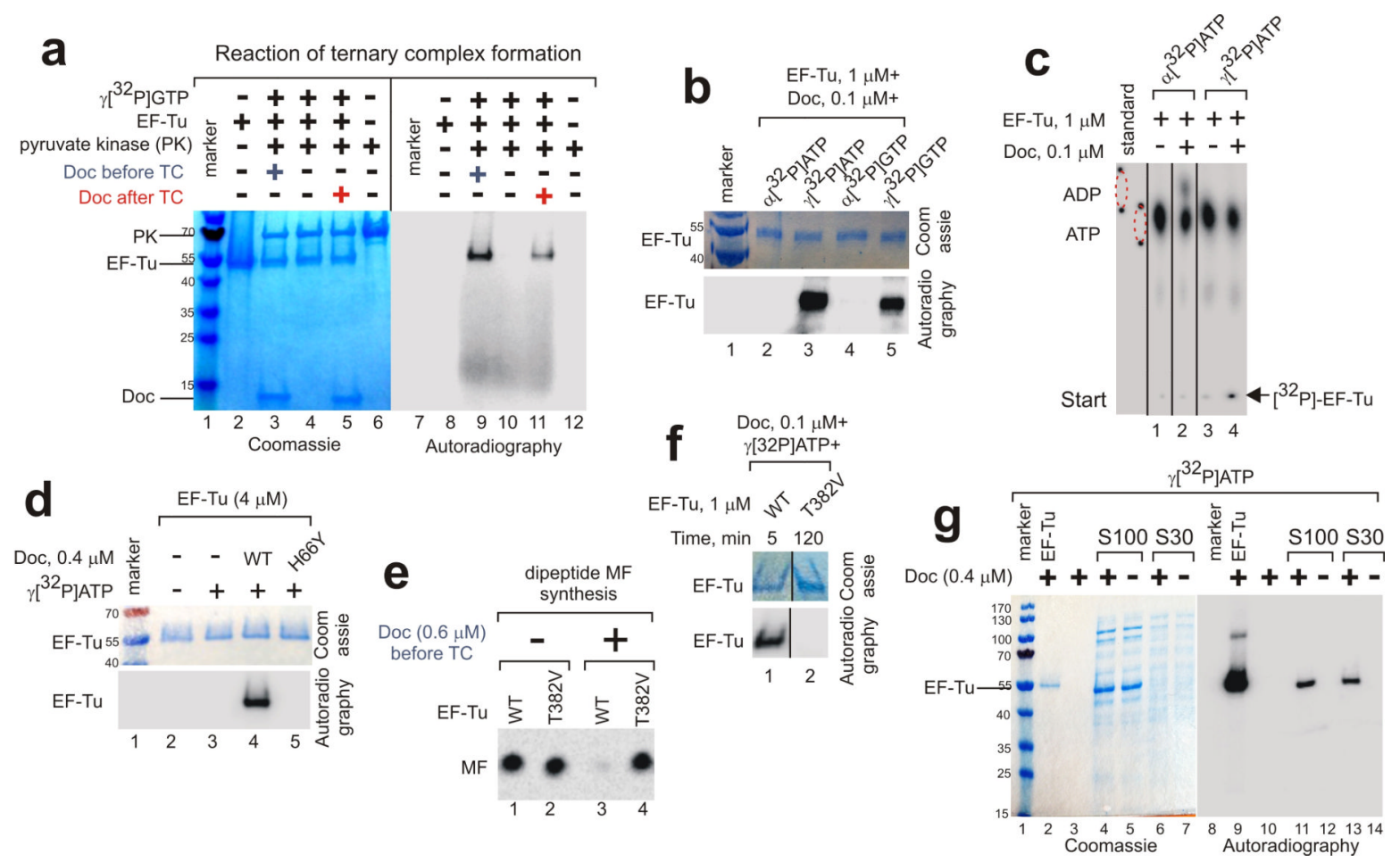

Figure 2. Doc phosphorylates EF-Tu at the conserved Thr382

(a). Radiolabeling of EF-Tu by Doc added during (blue) or after (for $15 \mathrm{~min}$; red) ternary complex formation in the presence of $\mathrm{Y}\left[{ }^{32} \mathrm{P}\right]$-GTP (here and after 3 pmol unless otherwise specified) was analyzed by SDS-PAGE and autoradiography. (b). Radiolabeling of EF-Tu by Doc in the presence of $a\left[{ }^{32} \mathrm{P}\right]-\mathrm{ATP}, \mathrm{y}\left[{ }^{32} \mathrm{P}\right]-\mathrm{ATP}, \mathrm{a}\left[{ }^{32} \mathrm{P}\right]-\mathrm{GTP}$ or $\mathrm{Y}\left[{ }^{32} \mathrm{P}\right]-\mathrm{GTP}$. (c). Products of EF-Tu modification $(30 \mathrm{~s})$ by Doc in the presence of $\mathrm{a}\left[{ }^{32} \mathrm{P}\right]-\mathrm{ATP}$ or $\mathrm{\gamma}\left[{ }^{32} \mathrm{P}\right]-\mathrm{ATP}$ were separated by thin layer chromatography (PEI-cellulose in $0.5 \mathrm{M} \mathrm{K}_{2} \mathrm{HPO}_{4}$ ) and analyzed by autoradiography. ATP and ADP mobility standards were visualized under UV25435,36 and marked with radioactive spots before phosphorimaging. (d). Phosphorylation of EF-Tu by catalytic mutant of Doc, H66Y. (e). Dipeptide MF synthesis with EF-Tu and EF-Tu ${ }^{\mathrm{T} 382 \mathrm{~V}}$ in the absence or presence of Doc added before ternary complex formation. (f). Phosphorylation of wild-type EF-Tu and mutant EF-Tu ${ }^{\mathrm{T} 382 \mathrm{~V}}$. (g). Doc and $\mathrm{\gamma}\left[{ }^{32} \mathrm{P}\right]-\mathrm{ATP}$ were added to purified EF-Tu, S30 or S100 lysate fractions. A band migrating above the purified EF-Tu corresponds to in vitro aggregated EF-Tu ${ }^{37}$. 

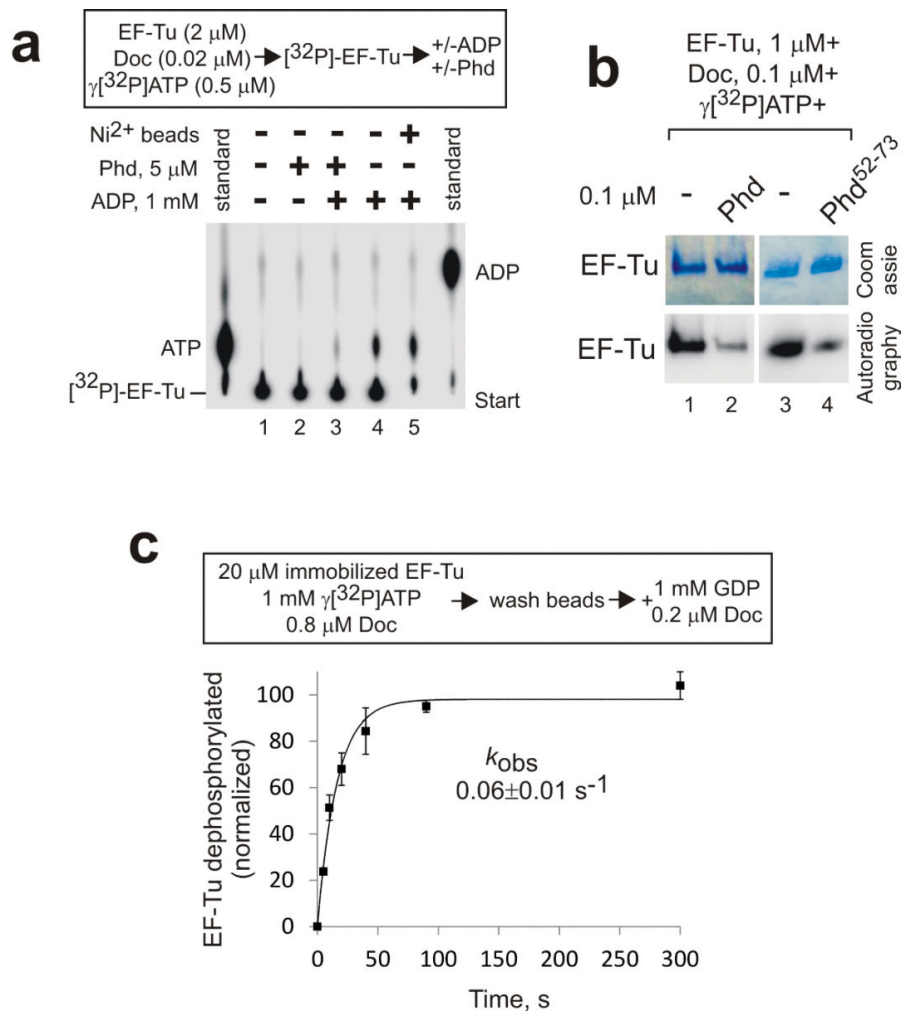

Figure 3. Inhibition of phosphorylation of EF-Tu and its dephosphorylation

(a). A scheme of the experiment is shown above the radiogram (see also Supplementary Fig. 6). EF-Tu ${ }^{32} \mathrm{P}$-phosphorylated by Doc (ensuring full usage of $\mathrm{y}\left[{ }^{32} \mathrm{P}\right]-\mathrm{ATP}$ ) was then incubated with or without Phd and/or ADP. The products were analyzed by TLC. For ATP mobility standard the reaction of EF-Tu phosphorylation in the presence of $\mathrm{Y}\left[{ }^{32} \mathrm{P}\right]-\mathrm{ATP}$ was spotted on the TLC plate immediately after start of the reaction. For ADP mobility standard $\mathrm{a}\left[{ }^{32} \mathrm{P}\right]-\mathrm{ATP}$ was used in the reaction of EF-Tu phosphorylation, which resulted in formation of $\mathrm{a}\left[{ }^{32} \mathrm{P}\right]-\mathrm{ADP}$. Nonradioactive standards, visualized under UV254 are not displayed. Not all EF-Tu can be dephosphorylated even after prolonged incubation either due to aggregation or to the competition from phosphorylation with the $\mathrm{V}\left[{ }^{32} \mathrm{P}\right]$-ATP produced in the reaction. The identity of the of ${ }^{32} \mathrm{P}$-phosphorylated His-tagged EF-Tu on TLC was verified by addition of $\mathrm{Ni}^{2+}$-NTA-agarose to the reaction. (b). Phosphorylation of EF-Tu by Doc was performed in the absence and presence of equimolar amounts of Phd or its toxin-binding domain Phd ${ }^{52-73}$. (c). Kinetics of dephosphorylation of immobilized ${ }^{32} \mathrm{P}$-phosphorylated EF$\mathrm{Tu}$ (see Online Methods) in the presence of GDP and Doc. Data are the mean of three independent experiments and error bars show standard deviations. Data were fitted into a single-exponential equation and normalized to the predicted maximum, which was taken as 100. \pm sign represents standard error of the fit. 



Figure 4. Solution structure of the Doc:EF-Tu:GDP complex by SAXS

Experimental SAXS scattering curves (grey) for Doc (a), EF-Tu:GDP (b) and Doc:EFTu:GDP (c) overlaid with computed scattering curves obtained from rigid body modeling (black) of the particles (see Online Methods for details). Bars are experimental errors of the measurements. The rigid body reconstructed models of the EF-Tu:GDP complex (d) and the Doc:EF-Tu:GDP ternary complex (e) are superimposed onto the $a b$ initio envelopes of the complexes reconstructed from the experimental SAXS data using the program DAMMIF from the ATSAS suite ${ }^{23}$ (see also Supplementary Fig. 8). All the $a b$ initio models of the complex were very reproducible in independent runs with average normalized spatial discrepancy (NSD) values below 0.9. (f). ${ }^{1} \mathrm{H} /{ }^{15} \mathrm{~N}$ HSQC spectra of the titration of Doc (0 $\mu \mathrm{M}$ of EF-Tu in blue) with EF-Tu (34.0 $\mu \mathrm{M}$ (in purple), $58.0 \mu \mathrm{M}$ (in red), $123.3 \mu \mathrm{M}$ (in yellow) and 197.3 $\mu \mathrm{M}$ (in green) (see also Supplementary Fig. 7a, b). (g) and (h). $\mathrm{I} / \mathrm{I}_{\mathrm{O}}$ changes above $2 \sigma$ (green line) are colored blue on the surface of Doc (pdbid 3K33) (see also Figure 5c). (i) Doc (red ribbons) binds to "open" EF-Tu (blue surface; pdbid $1 \mathrm{EFC}^{21}$ ) in a central cavity (in purple) of $\sim 16 \AA$ formed between the G-domain and the two $\beta$-barrels domains. (j). Phe-tRNA ${ }^{\text {Phe }}$ from the ternary complex (pdbid 1TTT ${ }^{22}$ ) superimposed on the model of the Doc:Tu:GDP complex. Notice that CCA tail of tRNA (in yellow) overlaps with the binding site of Doc. 



Figure 5. Chemical shift mapping of the nucleotide and EF-Tu binding sites of Doc

(a) ${ }^{1} \mathrm{H} /{ }^{15} \mathrm{~N}$ HSQC spectra of Doc (in blue) titrated with AMPPNP (1.4 mM (purple), 2.7 $\mathrm{mM}$ (red), $9.0 \mathrm{mM}$ (brown), $15.0 \mathrm{mM}$ (orange), $25.8 \mathrm{mM}$ (yellow) and 40.0 mM (green)). (b) Residue specific AMPPNP-induced chemical shift changes of Doc. (c) Mapping on the surface of Doc of residues (colored raspberry) with chemical shift perturbations $(\Delta \delta)>2 \sigma$ (green line in panel b) and residues that disappear upon AMPPNP binding (colored salmon). (d) Chemical shift based docking model of the Doc:AMPPNP complex. Doc active site loop is highlighted in red and AMPPNP is shown as spheres (see also Supplementary Fig. 7d and 9). 




Figure 6. Proposed catalytic mechanism of the Doc-type Fic kinases

(a). The active site architecture and transposed binding of the NTP (shown in orange) led us to propose a catalytic mechanism used by Doc that, as in the case of other Fic proteins, involves the highly conserved histidine as the general base. The $\alpha$ - and $\beta$-phosphates of the NTP are most likely stabilized by the side chains of the conserved Lys73 and Arg74 residues of the catalytic loop. His66 hydrogen bonds (represented as a dotted line in both panels) the attacking hydroxyl group from Thr382 (in blue) and activates the oxygen atom for the nucleophilic attack. The catalytic residues from Doc are shown in raspberry. (b). Cartoon representation of the general reaction mechanism for Doc (left) and Fic (right) proteins. The reorientation of the NTP in the active site enables transferring the $\mathrm{Y}$-phosphate instead of the NMP moiety while maintaining the active site chemistry. Note, that the targets are shown schematically only for representation purposes. 